\title{
Real-time applications of Electron Cyclotron Emission interferometry for disruption avoidance during the plasma current ramp-up phase at JET
}

\author{
M. Fontana, ${ }^{1}$, a) C. D. Challis, ${ }^{2}$ N. J. Conway, ${ }^{2}$ R. Felton, ${ }^{2}$ A. Goodyear, ${ }^{2}$ C. Hogben, ${ }^{2}$ A. Peacock, ${ }^{3}$ S. \\ Schmuck, ${ }^{4}$ and JET Contributors ${ }^{\mathrm{b})}$ \\ ${ }^{1)}$ Ecole Polytechnique Fédérale de Lausanne (EPFL), Swiss Plasma Center (SPC), CH-1015 Lausanne, \\ Switzerland \\ ${ }^{2)}$ CCFE, Culham Science Centre, Abingdon, OX14 3DB, UK \\ 3) JET Exploitation Unit, Culham Science Centre, Abingdon, OX14 3DB, UK \\ ${ }^{4)}$ Istituto per la Scienza e Tecnologia dei Plasmi, CNR, via Cozzi 53, 20125 Milan, \\ Italy
}

(Dated: 9 July 2020)

Data produced by an electron cyclotron interferometer diagnostic are now available to the real-time control systems of of the joint European torus (JET) tokamak. The data consist of absolutely calibrated electron temperature profiles, covering the plasma low-field side, core and part of the high-field side, for most of the range of magnetic fields used at JET. The profiles are obtained without the need for real-time equilibrium reconstructions. A simple and robust metric for the peakedness of the profiles was defined and employed in a real-time control system. The main goal was to identify discharges at risk of disruption, after impurity accumulation in the core, and terminate them safely. This was successfully tested on the current ramp-up phase of hybrid plasmas, developed in support of high-performance scenarios for the upcoming deuteriumtritium JET campaign.

\section{INTRODUCTION}

An experimental campaign using deuterium-tritium (D-T) fuel, equivalent to what is foreseen will be used in a fusion reactor, is currently scheduled for 2021 on the joint European torus (JET) tokamak ${ }^{1}$. It will be, in fact, the first time that experiments with reactor-compatible fuel will be performed in a tokamak in the presence of ITER-like plasma facing components ${ }^{2,3}$ (beryllium walls and tungsten divertor). High-performance and ITERrelevant scenarios will constitute the core of the D-T campaign and they will require highly integrated realtime control systems in order to achieve quasi-stationary conditions $^{4,5}$. For example, in certain ITER-relevant scenarios, it was found that temperature hollowing during the ramp-up phase can lead to the formation of modes inside the plasma ${ }^{6}$. This severely limited the performance of the discharges and, at times, led to plasma disruptions. Particularly in the frame of a D-T campaign, being able to promptly detect this process and safely terminate these discharges would mitigate the risk of potentially dangerous disruptions. Furthermore, with D-T plasmas, strict budgets will be enforced both on the available tritium fuel and on the integrated flux of high energy neutrons generated by the fusion reactions. Hence, the identification and early termination of pulses that are not going to reach high-performance conditions could reduce nuclear fuel consumption.

Diagnostics feeding pre-processed data to real-time control systems have to satisfy a long list of often com-

\footnotetext{
a)matteo.fontana@epfl.ch

b) See the author list of E. Joffrin et al. 2019 Nuclear Fusion 59 112021, https://doi.org/10.1088/1741-4326/ab2276
}

peting requirements, varying considerably with specific applications. For example, multiple electron temperature $\left(T_{e}\right)$ diagnostics are normally included in the realtime control systems of high-temperature plasma experiments. Two common choices are electron cyclotron emission (ECE) radiometers ${ }^{7-10}$ and Thomson scattering (TS) spectrometers ${ }^{11-13}$. ECE radiometers can provide measurements with high temporal resolution $(>100$ $\mathrm{kHz}$ ), particularly useful for detecting MHD modes developing inside the plasma. TS diagnostics, instead, are often used to robustly monitor temperature and density profiles with lower temporal resolution $(<100 \mathrm{~Hz})$ but independently from the plasma equilibrium reconstruction and without the risk of reaching cut-off conditions in high density or low magnetic field pulses ${ }^{14}$. At JET both the ECE radiometer ${ }^{15,16}$ and the high resolution TS (HRTS) ${ }^{17,18}$ have been successfully used in real-time control systems ${ }^{1920}$.

This paper presents the first real-time applications of the JET extraordinary mode (X-mode) ECE interferometer ${ }^{21,22}$. This diagnostic provides $T_{e}$ profiles with moderate time resolution $(60 \mathrm{~Hz})$. The ECE interferometer is absolutely calibrated over a wide range of frequencies $(70-500 \mathrm{GHz})$, independently from other diagnostics. Thus, its profiles cover the plasma low-field side, core and part of the high-field side, for most of the magnetic field values accessible to JET (1.4-3.8 T). It is only stopped from providing full $T_{e}$ profiles in pulses in which a combination of low magnetic field and/or high density cause cut-off conditions for ECE emission to arise ${ }^{14}$. The basic characteristics and principles of the JET ECE interferometer will be briefly presented in section II, focusing on the most relevant details for the real-time system, such as the basic hardware components and the data acquisition characteristics. 
Real-time applications for ECE interferometry are completely novel. This is because ECE radiometers have much higher temporal resolution $(>100 \mathrm{~Hz}$ for ECE radiometers) and simpler data processing. After applying a suitable calibration, in fact, the voltage output of radiometers channels immediately translates to electron temperature at specific radial positions. ECE interferometers, instead, produce interferograms that need to be separated and processed in order to get information about the ECE spectrum. On the other side, though, the independent, absolute calibration of the JET ECE interferometer and the large spectral coverage of the diagnostic make it an interesting choice for certain real-time applications. Section III will present the architecture of the real-time ECE interferometry system that was developed and its connections to the real-time data network of JET. Furthermore, the approximations contained in the real-time data processing and their influence on the resulting profiles will be discussed.

One of the applications that motivated the development of real-time ECE interferometry is the estimation of the hollowing of core $T_{e}$ during the initial ramping phase of high-performance plasma scenarios. A simple metric to define the peakedness of the electron temperature profile, independent from the equilibrium reconstruction, was defined to produce results robustly and with minimum delay, starting from ECE interferometer measurements. A control system based on this metric was developed and tested on discharges realized during the 2019 deuterium campaign on JET. Plasmas presenting temperature hollowing during the ramp-up phase were safely terminated by the control system. This first application and the results that were obtained will be described in details in Section IV.

Finally, conclusions and outlooks for real-time ECE interferometry at JET will be presented in section $\mathrm{V}$.

\section{X-MODE JET ECE INTERFEROMETER}

\section{A. Basic hardware and diagnostic principle}

The JET ECE interferometry system is used to provide absolutely calibrated electron temperature profiles over a large fraction of the plasma radial profile. It consists of two Martin-Puplett interferometers, looking at emission in fundamental ordinary mode (MI-O) and secondharmonic extraordinary mode (MI-X) respectively. Only MI-X is currently connected to the real-time data network of JET, so the remainder of the paper will treat exclusively the $\mathrm{X}$-mode interferometer. A detailed description of the instrument (including the calibration procedure) can be found in previous publications ${ }^{21,22}$. Here only the information most relevant to the new real-time system will be reported.

Radiation from the plasma is collected by a horizontal rectangular horn antenna $(50 \times 65 \mathrm{~mm})$ placed $0.353 \mathrm{~m}$ above the vessel mid plane, looking perpendicular to the

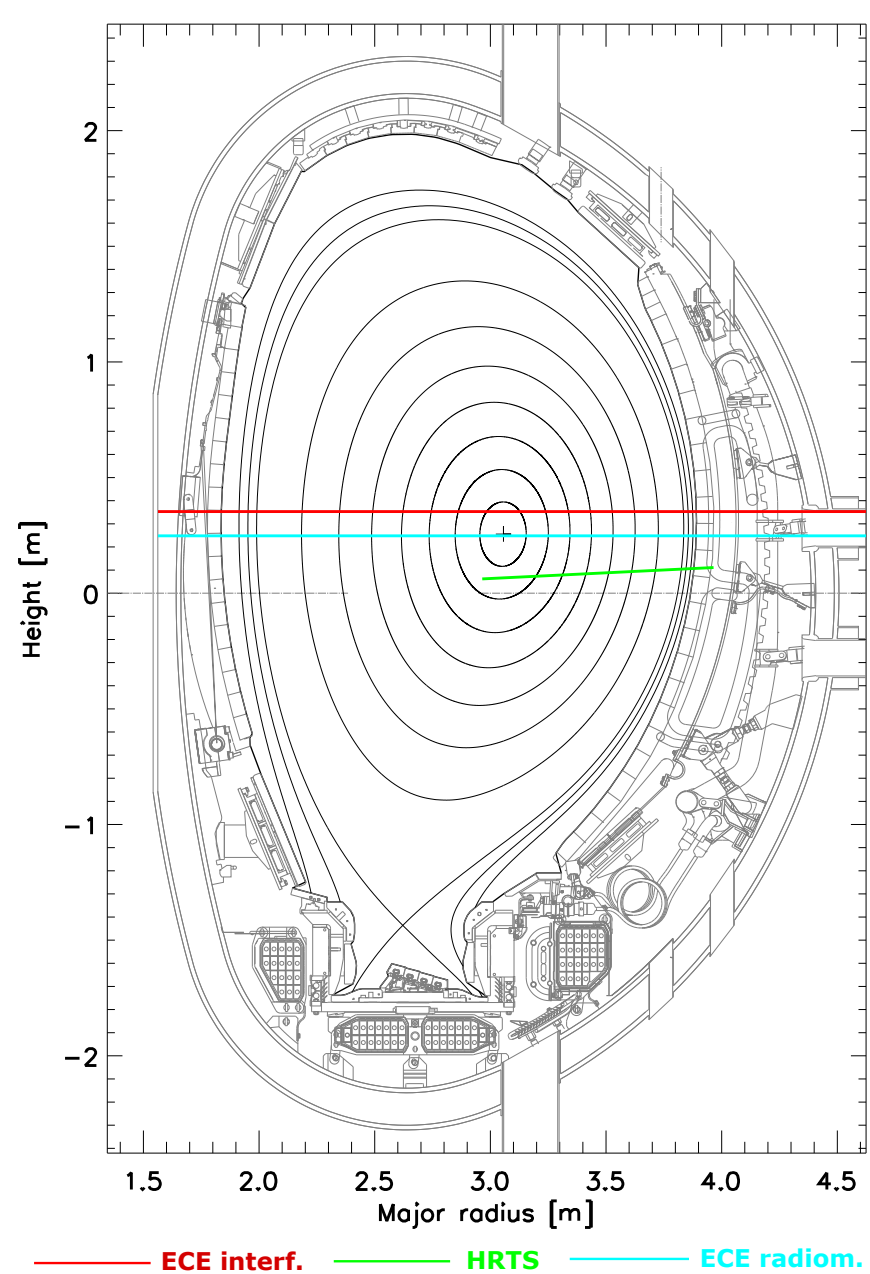

FIG. 1: Flux surfaces for a JET pulse (94945) at $11 \mathrm{~s}$, as calculated by EFIT. In the poloidal cross-section the lines of sight of the X-mode ECE interferometer ECE radiometer and high resolution Thomson scattering are displayed.

magnetic filed lines. The resulting line of sight of the instrument, together with those for the ECE radiometer and HRTS, are represented over a poloidal cross-section of the JET vessel in figure 1 . In the rectangular waveguide, immediately outside the vacuum window sealing the vessel access, a horizontal wire grid selects X-mode polarization. The radiation is carried for forty metres, in air-filled rectangular waveguides, to the interferometer in the diagnostic lab.

A simplified schematic of the interferometer is presented in the top left corner of figure 2. The incoming EC radiation from the plasma is split equally, by a wire grid polariser, into two perpendicular arms, each one terminating with a rooftop mirror that rotates the polarization by $90^{\circ}$. The two beams recombine at the polariser and are reflected into a cryogenically cooled InSb detector (QMC instruments MD1865). The detector is biased with a constant current, and the incoming power changes its resistivity. The resulting voltage variations are then 


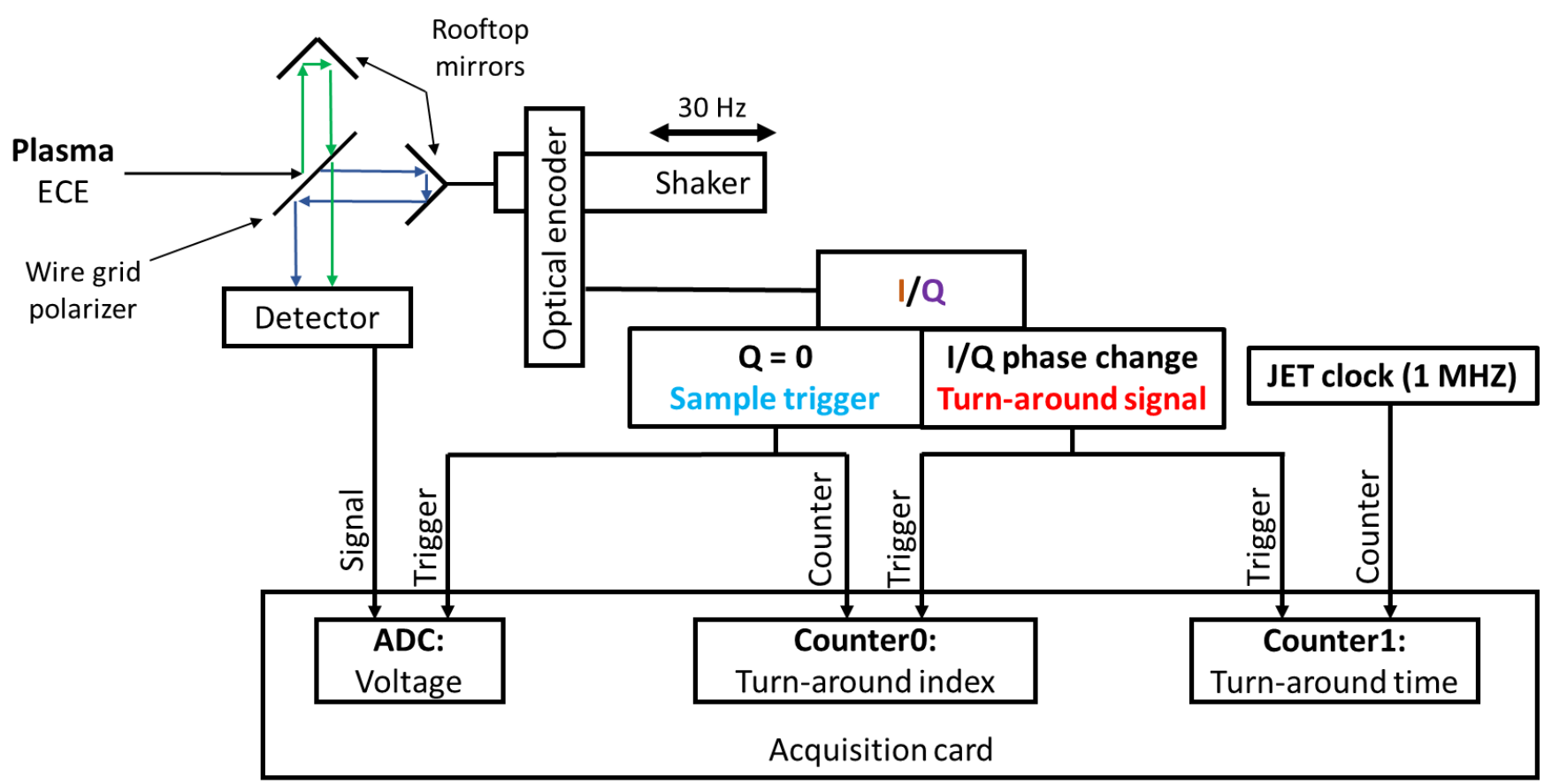

FIG. 2: Simplified schematics of the ECE interferometer in JET and its data acquisition scheme.

amplified, low pass filtered at $30 \mathrm{kHz}$ and acquired by a National Instruments data acquisition card (NI PCI$6220)$. One of the rooftop mirrors is connected to a vibrating motor (Bruel \& Kjaer vibrator V406 M4) that makes it oscillate at $30 \mathrm{~Hz}$ with a peak-to-peak amplitude of $16 \mathrm{~mm}$. The phase difference between the waves travelling in the two different arms creates a time dependent interference pattern at the detector. According to the principles of Fourier spectroscopy ${ }^{22-25}$, the spectrum of the collected radiation can be obtained as the inverse Fourier transform of the interferogram $(V)$ as a function of the optical path difference $(x)$ between the two different arms of the instrument.

$$
V(x) \propto \int T_{r a d}(f) C_{T}(f) \cos \left(2 \pi \frac{f}{c} x\right) d f .
$$

If the plasma density is sufficiently high to be considered optically thick ${ }^{26}$, then the intensity of the cyclotron radiation is proportional to the radiative temperature $\left(T_{r a d}\right)$ of the emitting electrons ${ }^{27}$. A frequency dependent calibration factor $\left(C_{T}\right)$ is calculated on JET with in-vessel measurements of the spectrum of a known source ${ }^{21,22}$.

One interferogram is produced every half-cycle of the movable mirror $(16 \mathrm{~ms})$. The $3.66 \mathrm{GHz}$ frequency resolution of the resulting spectrum is limited by the $16 \mathrm{~mm}$ optical path difference probed during the translation.

In ECE diagnostic applications, knowing the magnetic field along the diagnostic line of sight, $B(R)$, allows one to associate each radial position to its corresponding EC emission frequency in the spectrum. For a radiometer or interferometer looking at second harmonic emission:

$$
f_{E C E}(R)=2 \frac{q_{e} B(R)}{2 \pi m_{e}},
$$

where $B$ is the magnetic field as a function of the radial coordinate $R$ and $q_{e}$ and $m_{e}$ are, respectively, the charge and mass of the electrons. In this way, a radial temperature profile is reconstructed from every interferogram sampled by the diagnostic. At JET, the frequency ranges used to determine profiles using the $\mathrm{X}$-mode interferometer can go from 70 to $200 \mathrm{GHz}$.

In conclusion, then, radial $T_{e}$ profiles are produced every $16 \mathrm{~ms}(60 \mathrm{~Hz})$, with a frequency resolution of 3.66 $\mathrm{GHz}$ corresponding to a resolution of $3-10 \mathrm{~cm}$ on the plasma radius depending on the local magnetic field gradient.

\section{B. Interferometer data acquisition}

A representation of the data acquisition architecture for the JET ECE interferometer is shown in figure 2.

Due to the constant frequency of the oscillating motor, a constant acquisition frequency at the detector would correspond to a sinusoidal spatial sampling of the interferograms. To acquire the interferograms linearly in space, an optical linear encoder (Heidenhain LIDA $19 / 40$ ) is fixed to the interferometer structure, looking over a graded scale $(20 \mu \mathrm{m}$ between each line). The scale, instead, is joined to the moving mirror. The encoder head contains a light source screened by a grating corresponding to the graded scale, and a photoelectric 


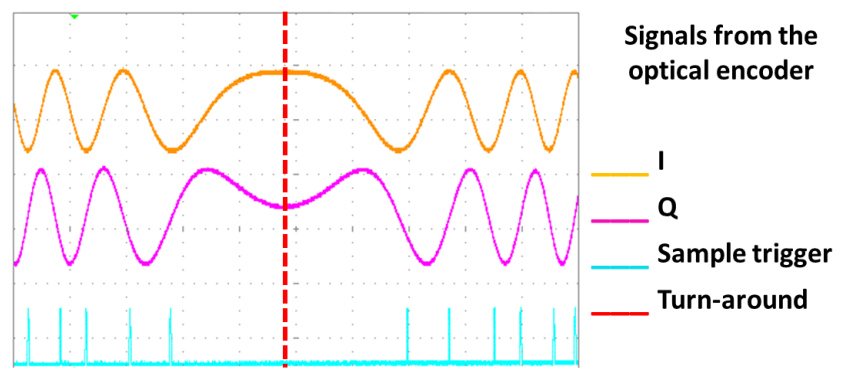

FIG. 3: Output signals from the linear encoder used for linear interferograms acquisition. A sample is recorded every time the $\mathrm{Q}$ output signal crosses 0 . The turn-around position is defined as the position in which the phase between the I and Q components changes sign from positive to negative.

detector. The latter produces an electrical signal modulated by the relative movement between the grating in front of the light source and the graded scale below it. The signals produced by the optical encoder in the $250 \mu \mathrm{s}$ around one of the mirror turn-around points are shown in figure 3. The I and Q components of the original signal are calculated and the positions at which $\mathrm{Q}=0$ are used to trigger the detector acquisition at fixed spatial displacements. In this way, each interferogram is acquired linearly with respect to the optical path difference between the two arms of the interferometers $(\Delta x=40$ $\mu \mathrm{m})$. The change of phase between the I and Q components (vertical dashed line in figure 3 ) identifies a turnaround point of the moving mirror. The indexes of the turn-around points corresponding to the rising edge of the phase change are recorded by a counter, in order to separate the consecutive interferograms during the signal processing. Simultaneously, another counter samples the corresponding turn-around time on the $1 \mathrm{MHz}$ JET clock. The turn-around counter, the turn-around time and the detector output are the three signals generated from the system.

\section{REAL-TIME ECE INTERFEROMETER SYSTEM: ARCHITECTURE, ACQUISITION AND PROCESSING}

\section{A. Real-time system architecture}

The data streaming architecture of the offline and realtime systems of the JET ECE interferometer are shown in figure 4 .

During a pulse, the three data streams (detector signal, turn-around indexes and time counters) are continuously passed by the acquisition card to the kernel memory of the acquisition PC. For the standard, off-line acquisition, the raw data are stored there until the end of the pulse, when they are sent by CODAS (control and data acquisition system) software to one of JET diagnostics subsystems as JET pulse files. There, they will be processed before the next plasma pulse.

As part of the real-time operation, instead, signals are processed locally in the acquisition PC. The way in which the real-time code selects the interferograms to process is illustrated in figure 5 . The process continuously looks for a new triggering of the turn-around index counter, represented in figure 5 by the dashed vertical lines. When a new index is found, the points acquired by the detector between the two latest recorded turn-arounds are divided in two halves and the second one is processed as a single interferogram (left side of figure 5). This produces the temperature profile corresponding to a single time point. The next interferogram is identified and processed when the same number of points is collected after the latest recorded turn-around index (right side of figure 5), generating the next time point in the signals. The code then waits for a new turn-around index to be recorded and the cycle then repeats.

The processing of the interferograms by the real-time algorithm is substantially identical to the procedure used for off-line data, described in detail in previous works ${ }^{22}$, with only a few differences. While the off-line code, running between discharges, is written in Python, the realtime code is written in C. It uses specialized libraries to optimize the discrete fast Fourier procedure necessary for the inverse transform of equation 1 , which is the most time consuming part of the processing. The running time of the code, for each interferogram, is about $1 \mathrm{~ms}$. The main difference between the real-time and off-line analysis is found in the profile reconstruction, after the calculation of the ECE spectra, and will be discussed in detail in the next section.

The outputs of the real-time code are transmitted via an ITER-like synchronous databus network ${ }^{28}$ (SDN), which is then bridged to the ATM (asynchronous transfer mode) interface of the JET real-time data network ${ }^{29}$, where they are available to the real-time central controller (RTCC). From there, they can be accessed by the real-time protection systems with a total of $2 \mathrm{~ms}$ delay. Each real-time signal is accompanied by a digital validation signal. This is used by the real-time system to distinguish times at which the result of the real-time calculations are not meaningful. This could be due, for example, to hardware faults.

\section{B. Approximations for real-time processing}

Since the magnetic equilibrium reconstruction is currently not available in real-time on JET, the value of the magnetic field along the line of sight of the ECE interferometer, $B(R)$ in equation 2, is approximated using only the measurement of the toroidal field coil current $\left(I_{t f c}\right)$. The formulas used to calculate the approximated magnetic field, $B_{\text {simple }}$, are:

$$
B_{\text {simple }}(R)=B_{\text {tor }}(R)=B_{\text {tor }}\left(R_{0}\right) \frac{R_{0}}{R} .
$$




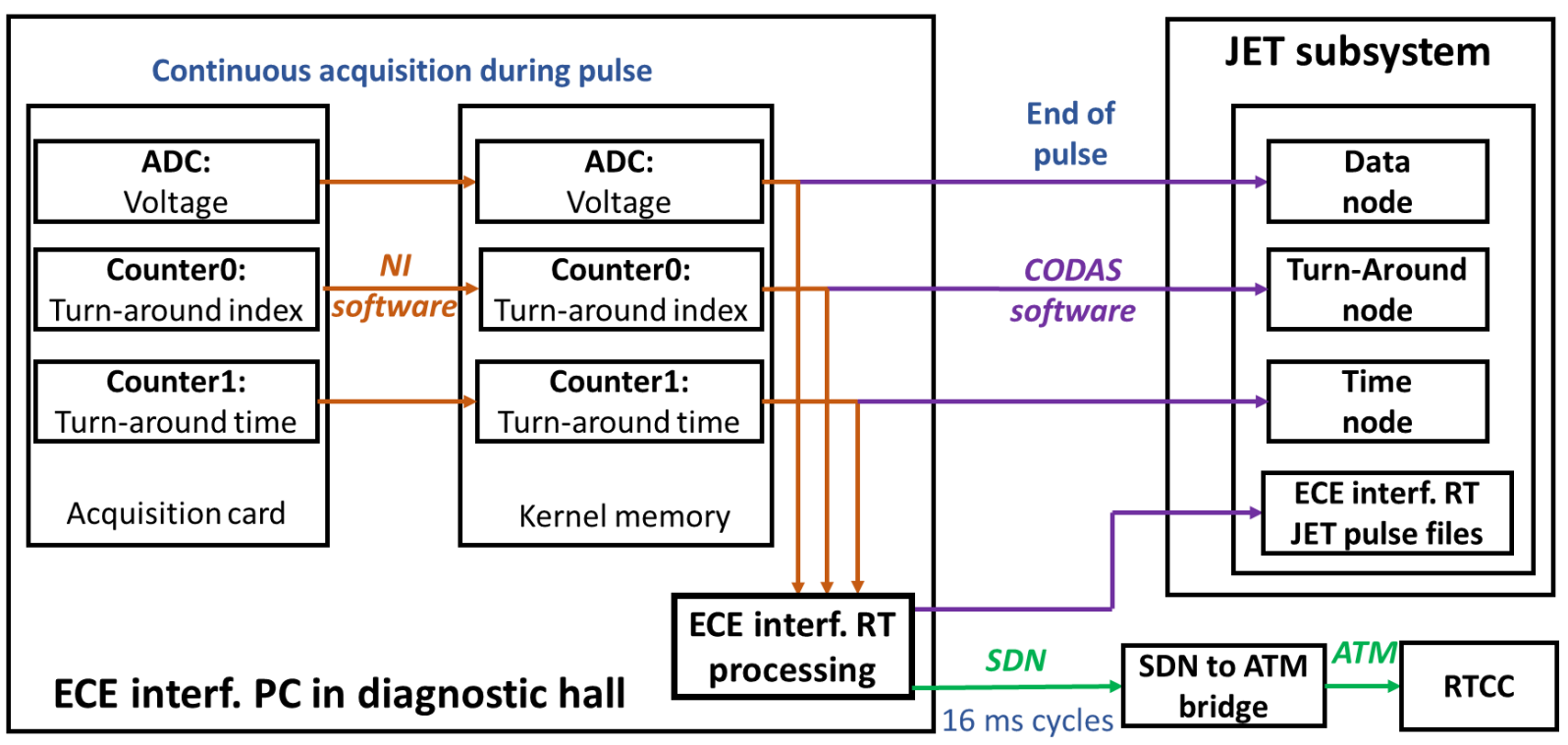

FIG. 4: Schematic representation of the acquisition architecture of the ECE interferometer and its connection with the real-time system.

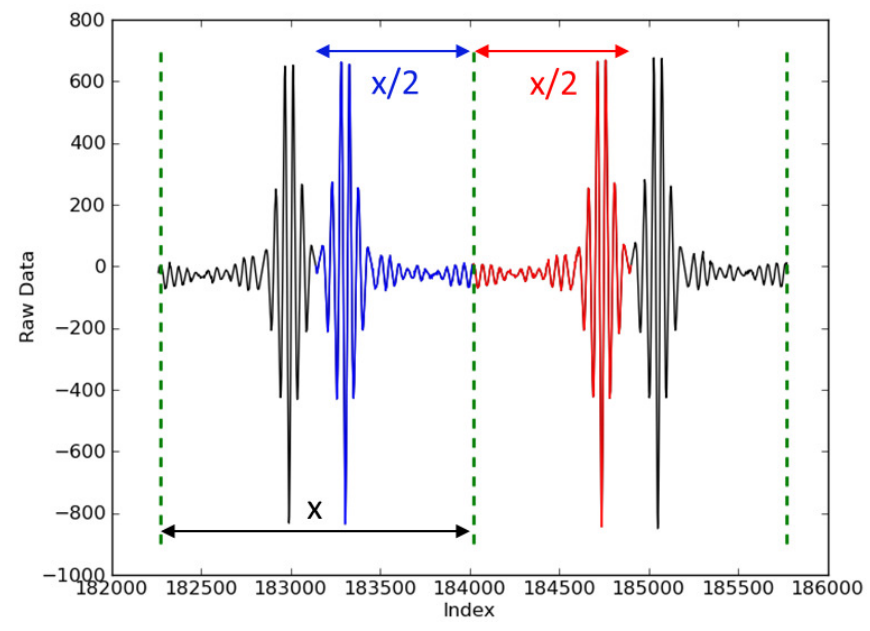

FIG. 5: Representation of four consecutive interferograms acquired around one of the recorded turn-around indexes.

$$
B_{\text {tor }}\left(R_{0}\right)=\frac{\mu_{0}}{2 \pi} \frac{I_{t f c}}{R_{0}} N_{\text {turns }} N_{\text {coils }} .
$$

In these equations, $B_{t o r}$ is the toroidal component of the magnetic field, $R_{0}$ the radial coordinate of the plasma axis, $\mu_{0}$ the magnetic permeability of vacuum and $N_{\text {turns }}$ is the number of turns inside each of the $N_{\text {coils }}$ toroidal field coils.

This approximation, in most cases, leads to underestimation of the total magnetic field, especially on the low field side, mainly due to the missing contribution of the poloidal field component. The error increases with the ratio between $I_{p}$ and $B_{t o r}$, making this approximation most appropriate for low current, high field pulses. Pro-

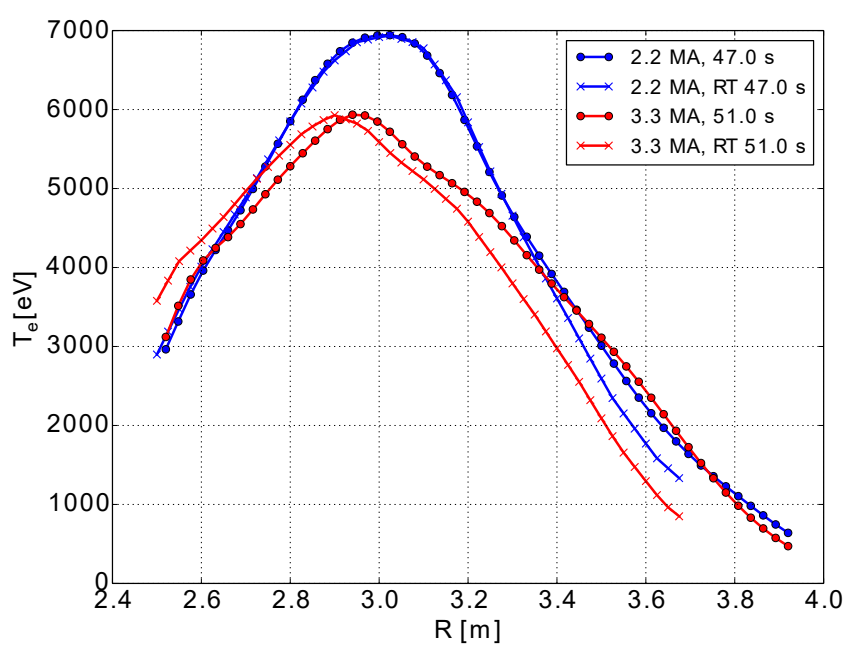

FIG. 6: Electron temperature profiles obtained with and without the magnetic field approximation used in the real-time system. Represented are one baseline plasma, with higher $I_{p}=3.3 \mathrm{MA}$ and $B_{t o r}=2.8 \mathrm{~T}$

(94788) and one hybrid plasma scenario with $I_{p}=2.2$ $\mathrm{MA}$ and $B_{\text {tor }}=2.8 \mathrm{~T}(95945)$.

files reconstructed with and without this approximation are shown in figure 6 . The error on the magnetic field is found to be $<4 \%$, for the flat-top of a baseline scenario plasma with $I_{p}=3.3 \mathrm{MA}$ and $B_{\text {tor }}=2.8 \mathrm{~T}$ (94788). This corresponds to inward shifts up to 5 and $15 \mathrm{~cm}$ at the plasma axis and at the LFS plasma edge respectively. However, in a plasma developed for the study of the hybrid scenario ${ }^{6}$, with by $I_{p}=2.2 \mathrm{MA}$ and $B_{t o r}=2.8$ $\mathrm{T}$ (94945), the deviation reduces to less than $3 \mathrm{~cm}$ for 
the whole profile. These errors are consistently smaller during the initial ramp-up phase, for all pulses.

Ideally, signals coming from other JET real-time systems could be used to estimate the other components of the magnetic field. However, this would add more dependencies to the real-time ECE calculations, increasing the chances of failure. For these first applications, a focus on simplicity and reliability was chosen. Currently, in fact, the only requirements for the real-time ECE calculations are ECE interferometry data and the measurement of the toroidal field coil current, making ECE real-time data very robust.

The signals produced by the real-time system must be used keeping in mind all the caveats normally employed for ECE measurements. For example, plasmas with very low density would have an optical depth too low to interpret the radiative temperature measured by ECE diagnostics as electron temperature. Similarly, plasmas too dense may present cut-off layers that stop ECE propagation and thus make measurements very complicated ${ }^{26}$.

\section{CORE TEMPERATURE HOLLOWNESS MONITORING USING ECE INTERFEROMETRY}

\section{A. Temperature hollowing in the ramp-up phase of hybrid discharges}

One of the main goals of the upcoming deuteriumtritium experimental campaign on JET is to test highperformance plasma scenarios with realistic nuclear fuel. Furthermore, it will be the first time that D-T plasmas are studied after the installation of the ITER-like wall on JET in 2011. Among these high-performance scenarios, are hybrid plasmas ${ }^{6,30}$, characterized by higher $\beta_{N}$ $\left(\beta_{N}=\frac{\langle p\rangle}{B_{t}^{2} / 2 \mu_{0}} \frac{a B_{T}}{I_{p}}\right)$ with respect to the baseline JET scenario. These plasmas usually start with a current ramp phase that is optimized in order to obtain a flat safety factor $(q)$ profile, with $q=1$ or slightly above it at the plasma core $\mathrm{e}^{30-32}$. This is achieved, on JET, using a fast current ramp that includes a current overshoot, with respect to the flat-top phase, before the start of the main heating systems ${ }^{6,30,33}$. Past experiments, studying the hybrid scenario with deuterium plasmas, observed the development of hollow electron temperature profiles during the ramp up phase, subsequent to core impurities accumulation and the consequent radiation. Some of these cases were followed by the formation of a $2 / 1$ double tearing mode that occasionally locked ${ }^{34}$, triggering the massive gas injection valve (MGI) of JET to end the pulse with a mitigated disruption ${ }^{33}$. Furthermore, even when the pulse did not disrupt, impurity accumulation can influence the q-profile. This is undesirable in plasma scenarios that are very sensitive to the safety factor profile shape. This was observed, in particular, after the installation of the metal ITER-like wall on JET ${ }^{35}$. In the frame of a D-T campaign, these events would substantially damage the scientific programme for two main reasons. First, a disrupted or dud discharge would be a waste of the limited budgets of tritium and neutron emission set for the experiments. Secondly, the presence of current overshoots in these discharges increases the risk of high current disruptions and, hence, of potential damage to the tokamak.

This was one of the main reasons that motivated the development of a real-time system able to reliably estimate electron temperature hollowing during the current ramp phase.

\section{B. Peakedness metric definition}

Starting from data provided by the ECE interferometer, a metric to estimate the temperature profile hollowness was developed with focus on speed, simplicity and robustness. Before the discharge, three radial windows are selected in the plasma profile and identified as core, left edge and right edge (highlighted for two specific pulses in figures $7 \mathrm{a}$ and $8 \mathrm{a}$ ). The position and width of the radial windows were selected empirically, by looking at the shape of the hollowing temperature profiles in the real-time interferometer signals. The temperature in the three windows is averaged, producing $T_{\text {core }}$ and $T_{\text {edge }}=\left(T_{\text {edge }, L}+T_{\text {edge }, R}\right) / 2$, then the peakedness metric $P$ was defined as:

$$
P=\frac{T_{\text {core }}-T_{\text {edge }}}{T_{\text {edge }}}
$$

The radial boundaries of the three radial windows can be modified in between pulses and multiple sets of windows can be defined as the same time (obtaining $P_{1}, P_{2}$ etc.), for example to optimize the calculation for different plasma phases, such as ramp-up and flat-top. It was, however, observed that the calculation was quite insensitive to small changes in the windows position and width.

This approach does not require any analysis to find the peaks and trough of the hollow profiles, since it is based on fixed radial windows. As a consequence, it results in a very quick calculation, that does not impact on the response time of the real-time system. Furthermore, this calculation is very robust: a result is always produced, provided that the interferometer is sending meaningful raw data.

\section{First applications and results}

One example of the disruptions mentioned in section IV A is shown in figures $7 \mathrm{a}$ and $7 \mathrm{~b}$ (pulse 94065). The electron temperature profiles shown in figure $7 \mathrm{a}$ are reconstructed by the ECE interferometer real-time system and the shaded regions correspond to the windows used to calculate $P_{1}$, as defined in equation 3 . There, it can be seen how, during the initial ramp-up phase, the electron temperature profile never becomes peaked and, around $3.5 \mathrm{~s}$ from breakdown, it starts hollowing. After reaching 


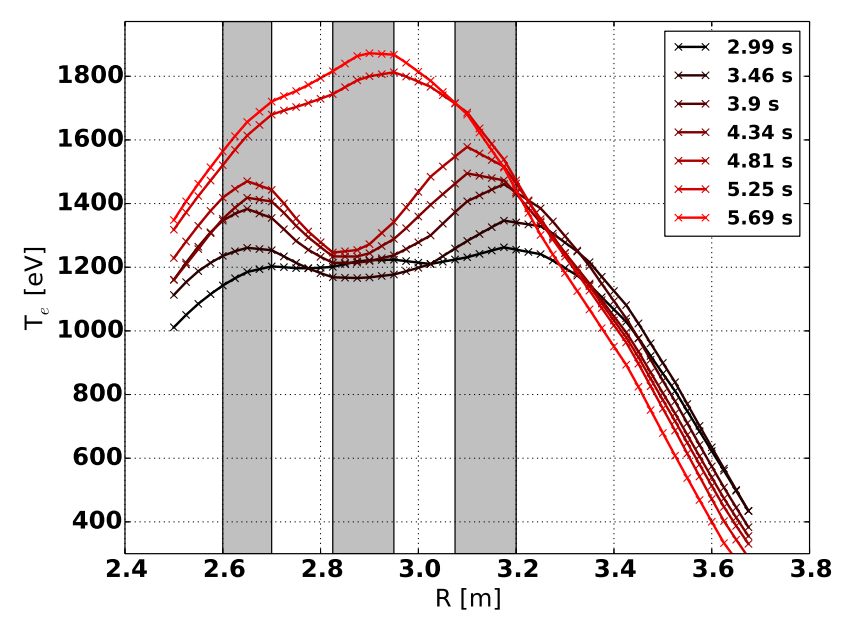

(a)

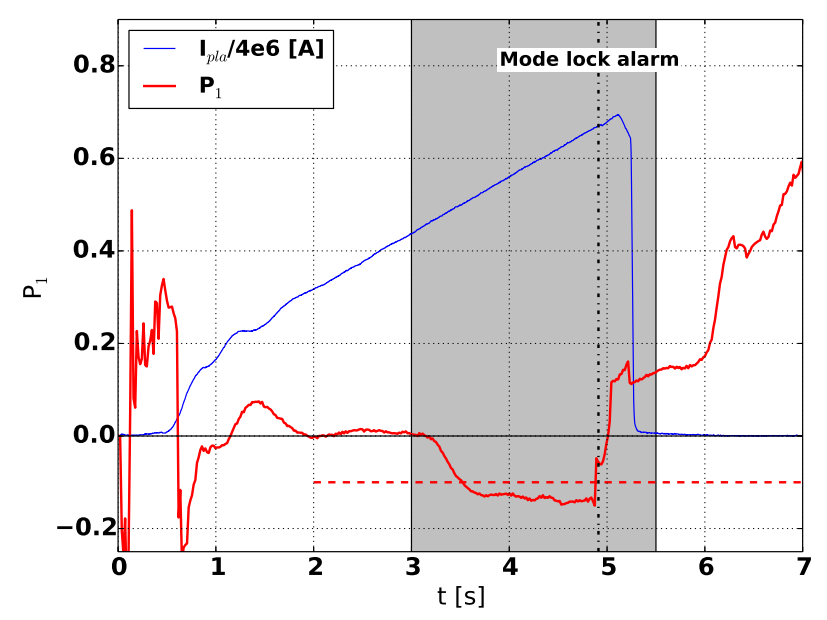

(b)

FIG. 7: (a) Electron temperature profiles of pulse 94065 up to its disruption. Highlighted are the radial windows used to estimate the profile peakedness as defined in equation 3. (b) Evolution of the plasma current and peakedness during the ohmic ramp up phase of the same pulse. The shaded area and the dashed red line represent the time interval and threshold that were chosen to define the control system for subsequent pulses. The vertical dash-dot line indicates the time at which a mode-lock alarm was sent to the real time protection system.

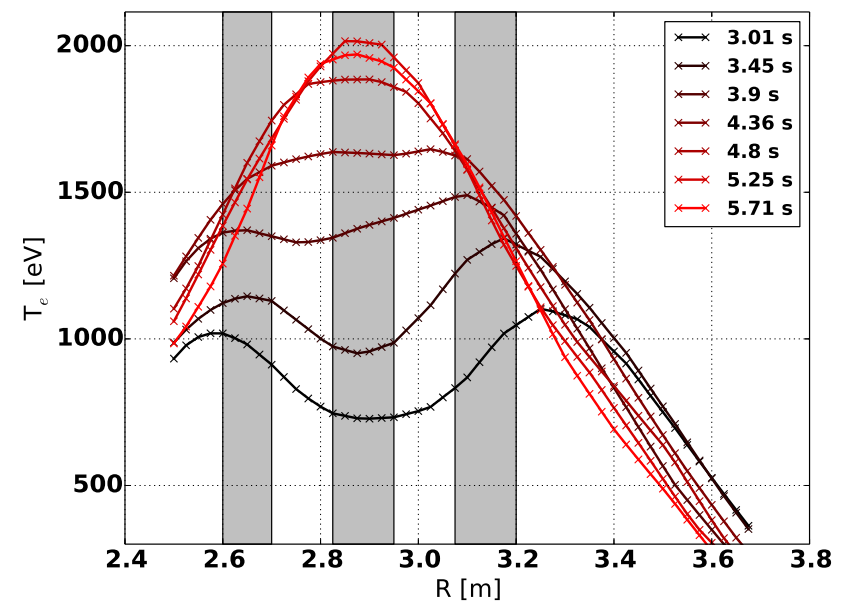

(a)

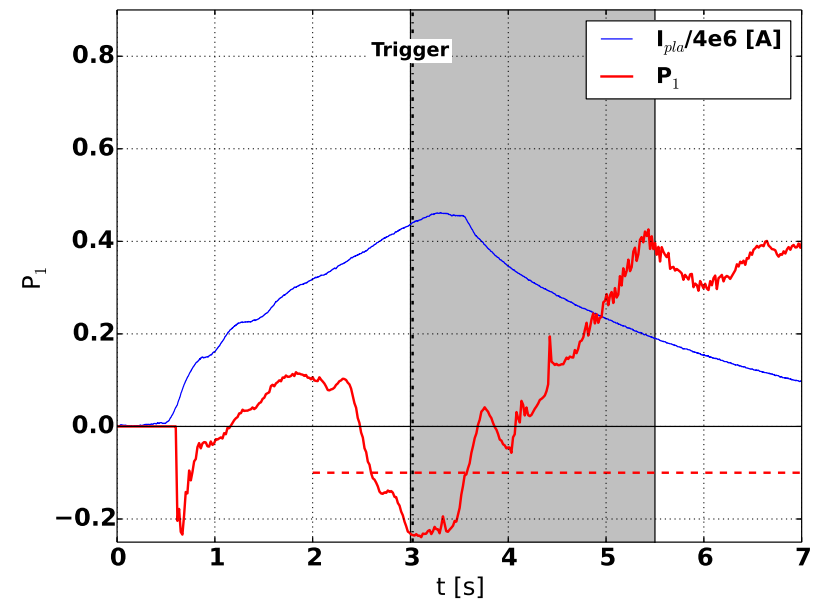

(b)

FIG. 8: (a) Electron temperature profiles of pulse 95252 during the initial ohmic current ramp (3-6 s). Highlighted are the radial windows used to estimate the profile peakedness as defined in equation 3. (b) Evolution of the plasma current and peakedness during the ohmic ramp up phase of the same pulse. Highlighted is the time region over which the controller is active. It sends a soft termination instruction when $P_{1}<-0.1$ for more than $20 \mathrm{~ms}$. In this case the system triggered at $3.022 \mathrm{~s}$ (vertical dash-dot line).

a critical value, a peaking profile is recovered but only for a very short time, after which the plasma disrupts. In figure $7 \mathrm{~b}$, the time evolution of the peakedness $P_{1}$ can be seen together with $I_{p}$. The peakedness reaches a low point around -0.15 at $\sim 3.5 \mathrm{~s}$. The profile remained in this state a little more than one second, then a locked mode was detected at $4.912 \mathrm{~s}$. After this, the profile evolved rapidly before the plasma eventually disrupted.

Based on this example and more cases from the 2016 JET campaign ${ }^{6,34}$, a simple control system was designed, looking for values of $P_{1}<-0.1$ for more than $20 \mathrm{~ms}$ in the time window [3-5.5] s (neutral beam heating would 
be turned on at $6 \mathrm{~s}$ ). When this condition is satisfied, a slow stop trigger is sent to the coils, the heating systems, the plasma density, shape and position controllers. This involves immediately turning off the gas supply and any form of auxiliary heating while, at the same time, ramping down the coils current over several seconds. The optical thickness of the plasma in the radial windows used to calculate $P_{1}$ was verified to be high enough $(>2)$ during the selected time window. This is to confidently interpret the radiative temperature measurements provided by the ECE diagnostic as electron temperature ${ }^{26}$.

The control system was tested on a series of hybrid pulses performed during the 2019 JET campaign. The intervention of the controller in one of these pulses (95252) is shown in figures $8 \mathrm{a}$ and $8 \mathrm{~b}$.

In figure $8 \mathrm{a}$ the electron temperature profiles, as calculated in real-time by the ECE interferometer, are shown for the whole controller activation window (3-5.5 s). The profiles show clearly a progressive flattening and hollowing, normally associated with impurity influx in the core. In this case, the radial windows used for calculating $P_{1}$ were not perfectly centred on the minimum and maxima of the hollow temperature profiles. However, the peakedness metric still captures well the hollowing process, as shown in figure $8 \mathrm{~b}$. There, the shaded area covers the times at which the the controller is active, and the dashed red line represents its threshold, set at -0.1 . In this case, the controller was immediately triggered (after $20 \mathrm{~ms}$ of assertion time) as soon as it turned on. This is shown by the vertical dash-dot line. The current ramp was then quickly stopped and the pulse proceeded with a soft termination.

Since August 2019, this control system has always been in place when performing hybrid pulses on JET. At the time of writing, all plasmas that triggered the control system were terminated successfully, without causing disruptions.

\section{CONCLUSIONS AND OUTLOOK}

Data produced by the X-mode ECE interferometer of JET, are now available to the tokamak real-time system. The diagnostic provides absolutely calibrated electron temperature profiles, reliably covering the plasma low field side and core for most of the magnetic field levels used at JET. The real-time ECE interferometer collects one interferogram every $16 \mathrm{~ms}$. Calculation of the temperature profiles takes less than $1 \mathrm{~ms}$, and $2 \mathrm{~ms}$ are necessary to transport the signal from the diagnostic PC to the real-time protection system. The main approximation, necessary to assure the speed of the calculations, is neglecting magnetic field components other than the vacuum toroidal field. This is especially relevant for pulses with high $I_{p}$ and low $B_{T}$ for which the effects are clearly evident on the low field side profiles. These effects were observed to not be relevant in the context of the current application of the real-time system. The main applica- tion of real-time ECE interferometry, in fact, is the detection of $T_{e}$ profile hollowing during the ramp-up phase, especially in the hybrid plasma scenario.

For this, a simple, robust hollowness metric was defined and employed as part of a control system that was tested on hybrid plasmas during the 2019 JET campaign, in order to avoid dud pulses and conditions favourable for disruptions. Pulses that displayed excessive hollowness during their ramp-up phase were terminated with a soft stop that, in all cases, ended the plasma without a disruption. This control system is currently regarded as fundamental for the next phase of development of highperformance hybrid pulses in preparation of the upcoming D-T experimental campaign. In principle, other realtime actions could be considered, such as gas injection or central RF heating, with the goal of increasing pulse efficiency when nuclear fuel will be employed.

Other applications for real-time ECE interferometry data are being explored. Among these, the possibility of relating a decrease in the outer core electron gradient to the onset of MHD modes often observed to precede disruptions in various high-performance scenarios.

Work is ongoing at JET to produce a real-time equilibrium reconstruction, based on EQUINOX ${ }^{36}$. When available, this will be integrated with the real-time ECE calculations in order to better take into account the full magnetic field in the profiles reconstruction.

The potential for real-time applications of ECE interferometry will be further explored in the coming months, with the possibility of connecting also the O-mode interferometer to the real-time data network. Combined data from both interferometers would allow to have, for a range of conditions where first harmonic O-mode emission is not in cut-off, profiles covering both the low and high field side.

\section{ACKNOWLEDGMENTS}

This work has been carried out within the framework of the EUROfusion Consortium and has received funding from the Euratom research and training programme 2014-2018 and 2019-2020 under grant agreement No 633053. The views and opinions expressed herein do not necessarily reflect those of the European Commission This work was supported in part by the Swiss National Science Foundation.

\section{DATA AVAILABILITY STATEMENT}

The data that support the findings of this study are available from the corresponding author upon reasonable request. 


\section{BIBLIOGRAPHY}

${ }^{1}$ X. Litaudon. JET Program for Closing Gaps to Fusion Energy. IEEE Transactions on Plasma Science, 44(9):1481-1488, September 2016.

${ }^{2}$ R. A. Pitts, S. Carpentier, F. Escourbiac, T. Hirai, V. Komarov, A. S. Kukushkin, S. Lisgo, A. Loarte, M. Merola, R. Mitteau, A. R. Raffray, M. Shimada, and P. C. Stangeby. Physics basis and design of the ITER plasma-facing components. Journal of Nuclear Materials, 415(1, Supplement):S957-S964, August 2011. ${ }^{3}$ R. A. Pitts, S. Carpentier, F. Escourbiac, T. Hirai, V. Komarov, S. Lisgo, A. S. Kukushkin, A. Loarte, M. Merola, A. Sashala Naik, R. Mitteau, M. Sugihara, B. Bazylev, and P. C. Stangeby. A full tungsten divertor for ITER: Physics issues and design status. Journal of Nuclear Materials, 438:S48-S56, 2013.

${ }^{4}$ R. Felton, E. Joffrin, A. Murari, L. Zabeo, F. Sartori, F. Piccolo, J. Farthing, T. Budd, S. Dorling, P. McCullen, J. Harling, S. Dalley, A. Goodyear, A. Stephen, P. Card, M. Bright, R. Lucock, E. Jones, S. Griph, C. Hogben, M. Beldishevski, M. Buckley, J. Davis, I. Young, O. Hemming, M. Wheatley, P. Heesterman, G. Lloyd, M. Walters, R. Bridge, H. Leggate, D. Howell, K. D Zastrow, C. Giroud, I. Coffey, N. Hawkes, M. Stamp, R. Barnsley, T. Edlington, K. Guenther, C. Gowers, S. Popovichef, A. Huber, C. Ingesson, D. Mazon, D. Moreau, D. Alves, J. Sousa, M. Riva, O. Barana, T. Bolzonella, M. Valisa, P. Innocente, M. Zerbini, K. Bosak, J. Blum, E. Vitale, F. Crisanti, E. de la Luna, and J. Sanchez. Real-time measurement and control at JET experiment control. Fusion Engineering and Design, 74(1):561-566, November 2005.

${ }^{5}$ M. Lennholm, I. S. Carvalho, K. Cave-Ayland, A. Chagnard, C. Challis, R. Felton, D. Frigione, L. Garzotti, A. Goodyear, J. P. Graves, C. Guillemaut, J. R. Harrison, E. Lerche, P. J. Lomas, R. Mooney, F. Rimini, A. C. C. Sips, C. Sozzi, D. Valcarcel, and J. Vega. Real time control developments at JET in preparation for deuterium-tritium operation. Fusion Engineering and Design, 123:535-540, November 2017.

${ }^{6}$ C. D. Challis, J. Garcia, M. Beurskens, P. Buratti, E. Delabie, P. Drewelow, L. Frassinetti, C. Giroud, N. Hawkes, J. Hobirk, E. Joffrin, D. Keeling, D. B. King, C. F. Maggi, J. Mailloux, C. Marchetto, D. McDonald, I. Nunes, G. Pucella, S. Saarelma, and J. Simpson and. Improved confinement in JET high $\$ \backslash$ upbeta $\$$ plasmas with an ITER-like wall. Nucl. Fusion, 55(5):053031, April 2015.

${ }^{7}$ N. K. Hicks, M. Garcia-Munoz, V. Igochine, M. Maraschek, M. Reich, J. Stober, W. Suttrop, and W. Treutterer. Real-time MHD Mode Localization in ECE Measurements on ASDEX Upgrade. APS, 51:GP8.034, November 2009.

${ }^{8}$ B. Esposito, G. Granucci, S. Nowak, J. R. Martin-Solis, L. Gabellieri, E. Lazzaro, P. Smeulders, M. Maraschek, G. Pautasso, J. Stober, W. Treutterer, L. Urso, F. Volpe, H. Zohm, and and and. Disruption control on FTU and ASDEX upgrade with ECRH. Nucl. Fusion, 49(6):065014, May 2009.

${ }^{9}$ B. Esposito, G. Granucci, M. Maraschek, S. Nowak, E. Lazzaro, L. Giannone, A. Gude, V. Igochine, R. McDermott, E. Poli, M. Reich, F. Sommer, J. Stober, W. Suttrop, W. Treutterer, H. Zohm, and and. Disruption avoidance by means of electron cyclotron waves. Plasma Phys. Control. Fusion, 53(12):124035, November 2011.

${ }^{10}$ H. van den Brand, W. A. Bongers, J. K. Stober, W. Kasparek, D. Wagner, N. Doelman, W. Klop, L. Giannone, M. Reich, E. Westerhof, M. R. de Baar, and and. Inline ECE measurements for NTM control on ASDEX Upgrade. Nucl. Fusion, 59(1):016013, December 2018.

${ }^{11}$ Sergei Shibaev, Graham Naylor, Rory Scannell, Graham J. McArdle, and Michael J. Walsh. Real Time Operation of MAST Thomson Scattering Diagnostic. IEEE Transactions on Nuclear Science, 58(4):1516-1521, August 2011.

${ }^{12}$ Florian Laggner, Egemen Kolemen, Ahmed Diallo, Benoit Leblanc, Roman Rozenblat, Greg Tchilinguirian, and NSTX-
U. Team Team. The real time multi point Thomson scattering diagnostic at NSTX-U. APS, 2017:PP11.068, October 2017.

${ }^{13} \mathrm{H}$. Arnichand, Y. Andrebe, P. Blanchard, S. Antonioni, S. Couturier, J. Decker, B. P. Duval, F. Felici, C. Galperti, P.-F. Isoz, P. Lavanchy, X. Llobet, B. Marlétaz, P. Marmillod, and J. Masur. New capabilities of the incoherent Thomson scattering diagnostics in the TCV tokamak: divertor and real-time measurements. J. Inst., 14(09):C09013-C09013, September 2019.

${ }^{14}$ L. Barrera, E. de la Luna, L. Figini, M. N. A. Beurskens, M. Brix, F. Castejón, P. C. de Vries, D. Farina, M. Kempenaars, P. Lomas, J. Mailloux, I. Nunes, and E. Solano and. Inboard and outboard electron temperature profile measurements in JET using ECE diagnostics. Plasma Phys. Control. Fusion, 52(8):085010, July 2010 .

${ }^{15}$ D. V. Bartlett, A. E. Costley, S. E. Jones, L. Porte, R. J. Smith, and A. Zolfaghari. The JET ECE heterodyne radiometer and investigations of fast phenomena. March 1993.

${ }^{16} \mathrm{E}$. de la Luna, J. Sánchez, V. Tribaldos, JET-EFDA contributors, G. Conway, W. Suttrop, J. Fessey, R. Prentice, C. Gowers, and J. M. Chareau. Electron cyclotron emission radiometer upgrade on the Joint European Torus (JET) tokamak. Review of Scientific Instruments, 75(10):3831-3833, October 2004.

${ }^{17}$ R. Pasqualotto, P. Nielsen, C. Gowers, M. Beurskens, M. Kempenaars, T. Carlstrom, and D. Johnson. High resolution Thomson scattering for Joint European Torus (JET). Review of Scientific Instruments, 75(10):3891-3893, October 2004.

${ }^{18}$ L. Frassinetti, M. N. A. Beurskens, R. Scannell, T. H. Osborne, J. Flanagan, M. Kempenaars, M. Maslov, R. Pasqualotto, and M. Walsh. Spatial resolution of the JET Thomson scattering system. Review of Scientific Instruments, 83(1):013506, January 2012 .

${ }^{19}$ D. Mazon, X. Litaudon, D. Moreau, M. Riva, G. Tresset, Y. Baranov, A. Bécoulet, J. M. Chareau, F. Crisanti, R. Dux, R. Felton, E. Joffrin, and contributors to the EFDA-JET workprogramme. Real-time control of internal transport barriers in JET $\backslash$ ast. Plasma Phys. Control. Fusion, 44(7):1087-1104, June 2002 .

${ }^{20}$ M. Zerbini, J.m. Chareau, D. Mazon, M. Riva, R. Felton, E. Joffrin, M. Lennholm, and R. Prentice. Fast ECE Diagnostics Applications For Real Time Control at JET. In Electron $C y$ clotron Emission and Electron Cyclotron Heating, pages 227232. WORLD SCIENTIFIC, February 2003.

${ }^{21} \mathrm{~S}$. Schmuck, J. Fessey, T. Gerbaud, B. Alper, M. N. A. Beurskens, E. de la Luna, A. Sirinelli, and M. Zerbini. Electron cyclotron emission measurements on JET: Michelson interferometer, new absolute calibration, and determination of electron temperature. Review of Scientific Instruments, 83(12):125101, 2012.

${ }^{22}$ S. Schmuck, J. Fessey, J. E. Boom, L. Meneses, P. Abreu, E. Belonohy, and I. Lupelli. Electron cyclotron emission spectra in Xand O-mode polarisation at JET: Martin-Puplett interferometer, absolute calibration, revised uncertainties, inboard/outboard temperature profile, and wall properties. Review of Scientific Instruments, 87(9):093506, September 2016.

${ }^{23} \mathrm{~J}$. W. Brault, George William Series, and Brian Arthur Thrush. Fourier transform spectrometry in relation to other passive spectrometers. Philosophical Transactions of the Royal Society of London. Series A, Mathematical and Physical Sciences, 307(1500):503-511, November 1982.

${ }^{24} \mathrm{~K}$. J. Button. Infrared and millimeter waves. Vol._6. Systems and components. 1982.

${ }^{25} \mathrm{C}$. D. Porter and D. B. Tanner. Correction of phase errors in fourier spectroscopy. Int J Infrared Milli Waves, 4(2):273-298, March 1983.

${ }^{26}$ M. Bornatici, R. Cano, O. De Barbieri, and F. Engelmann. Electron cyclotron emission and absorption in fusion plasmas. Nucl. Fusion, 23(9):1153, September 1983.

${ }^{27}$ G. Bekefi. Radiation Processes in Plasmas. 1966.

${ }^{28}$ L. Boncagni, C. Centioli, F. Iannone, C. Neri, M. Panella, L. Pangione, M. Riva, M. Scappaticci, V. Vitale, and L. Zaccarian. Synchronous Databus Network in ITER: Open source real-time net- 
work for the next nuclear fusion experiment. Fusion Engineering and Design, 83(2):504-510, April 2008.

${ }^{29} \mathrm{~J}$. Waterhouse, A. Stephen, and N. Petrella. Introduction of ITER CODAC relevant technologies on JET and MAST. In Indico for IAEA Conferences (Indico), May 2019.

${ }^{30}$ J. Hobirk, F. Imbeaux, F. Crisanti, P. Buratti, C. D. Challis, E. Joffrin, B. Alper, Y. Andrew, P. Beaumont, M. Beurskens, A. Boboc, A. Botrugno, M. Brix, G. Calabro \textquotesingle, I. Coffey, S. Conroy, O. Ford, D. Frigione, J. Garcia, C. Giroud, N. C. Hawkes, D. Howell, I. Jenkins, D. Keeling, M. Kempenaars, H. Leggate, P. Lotte, E. de la Luna, G. P. Maddison, P. Mantica, C. Mazzotta, D. C. McDonald, A. Meigs, I. Nunes, E. Rachlew, F. Rimini, M. Schneider, A. C. C. Sips, J. K. Stober, W. Studholme, T. Tala, M. Tsalas, I. Voitsekhovitch, and P. C. de Vries and. Improved confinement in JET hybrid discharges. Plasma Phys. Control. Fusion, 54(9):095001, July 2012.

${ }^{31}$ A. C. C. Sips, R. Arslanbekov, C. Atanasiu, W. Becker, G. Becker, K. Behler, K. Behringer, A. Bergmann, R. Bilato, D. Bolshukhin, K. Borrass, B. Braams, M. Brambilla, F. Braun, A. Buhler, G. Conway, D. Coster, R. Drube, R. Dux, S. Egorov, T. Eich, K. Engelhardt, H.-U. Fahrbach, U. Fantz, H. Faugel, M. Foley, K. B. Fournier, P. Franzen, J. C. Fuchs, J. Gafert, G. Gantenbein, O. Gehre, A. Geier, J. Gernhardt, O. Gruber, A. Gude, S. G. nter, G. Haas, D. Hartmann, B. Heger, B. Heinemann, A. Herrmann, J. Hobirk, F. Hofmeister, H. Hohen cker, L. Horton, V. Igochine, D. Jacobi, M. Jakobi, F. Jenko, A. Kallenbach, O. Kardaun, M. Kaufmann, A. Keller, A. Kendl, J.-W. Kim, K. Kirov, R. Kochergov, H. Kollotzek, W. Kraus, K. Krieger, B. Kurzan, P. T. Lang, P. Lauber, M. Laux, F. Leuterer, A. Lohs, A. Lorenz, C. Maggi, H. Maier, K. Mank, M.-E. Manso, M. Maraschek, K. F. Mast, P. McCarthy, D. Meisel, H. Meister, F. Meo, R. Merkel, D. Merkl, V. Mertens, F. Monaco, A. M. ck, H. W. M. ller, M. M. nich, H. Murmann, Y.-S. Na, G. Neu, R. Neu, J. Neuhauser, J.-M. Noterdaeme, I. Nunes, G. Pautasso, A. G. Peeters, G. Pereverzev, S. Pinches, E. Poli, M. Proschek, R. Pugno, E. Quigley, G. Raupp, T. Ribeiro, R. Riedl, S. Riondato, V. Rohde, J. Roth, F. Ryter, S. Saarelma, W. Sandmann, S. Schade, H.-B. Schilling, W. Schneider, G. Schramm, S. Schweizer, B. Scott, U. Seidel,
F. Serra, S. Sesnic, C. Sihler, A. Silva, E. Speth, A. St bler, K.H. Steuer, J. Stober, B. Streibl, E. Strumberger, W. Suttrop, A. Tabasso, A. Tanga, G. Tardini, C. Tichmann, W. Treutterer, M. Troppmann, P. Varela, O. Vollmer, D. Wagner, U. Wenzel, F. Wesner, R. Wolf, E. Wolfrum, E. W. rsching, Q. Yu, D. Zasche, T. Zehetbauer, H.-P. Zehrfeld, and H. Zohm. Steady state advanced scenarios at ASDEX Upgrade. Plasma Phys. Control. Fusion, 44(12B):B69-B83, November 2002.

${ }^{32}$ T. C. Luce, M. R. Wade, J. R. Ferron, A. W. Hyatt, A. G. Kellman, J. E. Kinsey, R. J. La Haye, C. J. Lasnier, M. Murakami, P. A. Politzer, and J. T. Scoville. Stationary high-performance discharges in the DIII-D tokamak. Nucl. Fusion, 43(5):321-329, April 2003.

${ }^{33}$ Clive D. Challis, Sebastijan Brezinsek, Ivor H. Coffey, Matteo Fontana, Nicholas Hawkes, David Keeling, Damian King, Gianluca Pucella, and Eleonora Viezzer. Effect of fuel isotope mass on q-profile formation in JET hybrid plasmas. Nucl. Fusion, 2020.

${ }^{34}$ L. Piron, T. C. Hender, E. Joffrin, F. Auriemma, P. Buratti, R. Paccagnella, P. Zanca, C. D. Challis, S. Gerasimov, R. Henriques, L. Marrelli, P. J. Lomas, G. Pucella, F. G. Rimini, and D. Terranova. Experimental and modelling study of locked mode dynamics prior to disruptions in high performance JET plasmas. 46 th EPS conference on plasma physics, 2019.

${ }^{35}$ J. Mailloux, C. Bourdelle, M. Baruzzo, P. Buratti, C. D. Challis, I. Coffey, E. de la Luna, P. De Vries, P. Gaudio, M. Gelfusa, N. Hawkes, J. Hobirk, I. Jenkins, E. Joffrin, V. Kiptily, M. Lehnen, P. J. Lomas, F. Orsitto, T. Putterich, C. Sozzi, and S. E. Sharapov. Impact of the JET ITER-like wall on the current ramp up phase and q-profile optimisation for hybrid nad advanced scenarios. 39th EPS conference on plasma physics, 2012.

${ }^{36}$ D. Mazon, J. Blum, C. Boulbe, B. Faugeras, M. Baruzzo, A. Boboc, S. Bremond, M. Brix, P. De Vries, S. Sharapov, L. Zabeo, and JET-EFDA Contributors. Equinox: a real-time equilibrium code and its validation at jet. In From Physics to Control Through an Emergent View, volume Volume 15 of World Scientific Series on Nonlinear Science Series B, pages 327-332. WORLD SCIENTIFIC, June 2010. 